\title{
Uso de la imagen radiológica y serología por Western Blot para el diagnóstico de la neurocisticercosis en un hospital del norte del Perú
}

\section{Use of radiological imaging and serology by Western Blot for the diagnosis of neurocysticercosis in a hospital in northern Peru}

Johnny Leandro Saavedra-Camacho, ${ }^{1, a}$, Mayra Massely Coico-Vega ${ }^{1, a}$, Virgilio E. Failoc-Rojas ${ }^{2, b}$, Benigno Ballón-Manrique ${ }^{3, c}$, Heber Silva-Díaz ${ }^{4,5, d}$

\section{RESUMEN}

Introducción: La neurocisticercosis (NCC) es una zoonosis parasitaria del sistema nervioso central causada por el céstodo Taenia solium, y que afecta a países en desarrollo y con escaso saneamiento básico. Objetivo: Describir el uso y la concordancia de la imagen radiológica por tomografía (TAC) o resonancia magnética (RM) y serología por western Blot (WB) en el diagnóstico de la NCC en un hospital del norte del Perú. Material y Métodos: Estudio observacional retrospectivo. La historia clínica fue la unidad de análisis. Los casos se buscaron en la oficina de Epidemiología mediante el CIE-10- B69 y registro del Laboratorio de Parasitología, Metaxénicas y Zoonosis del mismo hospital, en el periodo del año 2015 al 2017. Resultados: Se estudiaron 67 historias clínicas, que cumplían con los criterios diagnósticos absolutos de NCC. Los pacientes fueron varones en el 55,2 $\%$ y tuvieron una media de edad de $40,2( \pm 17,8)$ años. El $35,9 \%$ tuvieron un resultado positivo por WB (19/39), las lesiones quísticas con escólex fueron observada en el $25,4 \%$ de las TAC y en el 29,6 de las RM. La concordancia observada entre la prueba serológica con TAC y RM fue escasa, siendo (Kappa=0,073, IC95\%: 0,053 - 1,084) y (Kappa=0,112, IC95\%: 0,092 - 1,092) y un porcentaje de acuerdo de $42,0 \%$ y $45,7 \%$ respectivamente. Conclusiones: Se observó uso diferenciado y escasa concordancia entre la prueba serológica por WB e imagen radiológica en pacientes con diagnóstico de neurocisticercosis en la población estudiada.

Palabras Clave: Neurocisticercosis; Pruebas Serológicas; Diagnóstico por Imagen; Técnicas y Procedimientos Diagnósticos (Fuente: DeCSBIREME).

\section{ABSTRACT}

Background: Neurocysticercosis (NCC) is a parasitic zoonosis of the central nervous system caused by the tapeworm Taenia solium, which affects developing countries with poor basic sanitation. Objective: To describe the use and concordance of radiological tomography (CT) or magnetic resonance imaging (MRI) and western blot (WB) serology in the diagnosis of NCC in a hospital in northern Peru. Material and Methods: Retrospective observational study. The medical history was the unit of analysis. The cases were searched in the Epidemiology office using the ICD-10-B69 and registry of the Laboratory of Parasitology, Metaxenics and Zoonoses of the same hospital, in the period from 2015
FILIACIÓN

1. Facultad de Ciencias Biológicas, Universidad Nacional Pedro Ruiz Gallo. Calle Juan XXIII, Lambayeque, Perú.

Universidad San Ignacio de Loyola. Lima, Perú.

3. Servicio de Neurología, Hospital Regional Lambayeque. Chiclayo, Perú.

4. Facultad de Medicina Humana, Universidad San Martín de Porres. Chiclayo, Perú

5. Laboratorio de Parasitología, Metaxénicas y Zoonosis, Hospital Regional Lambayeque. Chiclayo, Perú.

a. Biólogo Microbiólogo.

b. Médico Cirujano, Maestro en Ciencias.

c. Médico Neurólogo.

D. Biólogo Microbiólogo, Doctor en Ciencias.

ORCID

1. Johnny Leandro Saavedra-Camacho

0000-0002-3842-4314

2. Mayra Massely Coico-Vega

0000-0001-8156-5449

Virgilio E. Failoc-Rojas

0000-0003-2992-9342

Benigno Ballón-Manrique

0000-0002-2073-4103

Heber Silva-Díaz
0000-0001-8263-9673

CORRESPONDENCIA

Johnny Leandro Saavedra-Camacho

Teléfono: +51990356090

EMAIL

johnnylsc107@gmail.com

CONFLICTOS DE INTERÉS

Los autores niegan conflictos de interés.

FINANCIAMIENTO

Autofinanciamiento.

\section{REVISIÓN DE PARES}

Recibido: 13/06/2021

Aceptado: 20/09/2021

\section{COMO CITAR}

Saavedra-Camacho, J., Coico-Vega, M., Failoc-Rojas, V., BallónManrique, B., \& Silva-Díaz, H. Uso de la imagen radiológica y serología por Western Blot para el diagnóstico de la neurocisticercosis en un hospital del norte del Perú. Revista Del Cuerpo Médico Hospital Nacional Almanzor Aguinaga Asenjo, 2021, 14(3), 311 - 315 . https://doi.org/10.35434/rcmhnaaa.2021.143.1251
Esta obra está bajo una Licencia Creative Commons Atribución 4.0 Internacional.

Versión Impresa: ISSN: 2225-5109

Versión Electrónica: ISSN: 2227-473

Cross Ref. DOI: $10.35434 /$ rcmhnaaa

OJS: https://cmhnaaa.org.pe/ojs 
to 2017. Results: 67 medicales records were studied, which complied with the absolute diagnostic criteria for NCC. The patients were men in $55.2 \%$ and had a mean age of $40.2( \pm 17.8)$ years. $35.9 \%$ had a positive result by WB (19/39), cystic lesions with scolex were observed in $25.4 \%$ of the CT and in 29.6 of the MRI. The concordance observed between the serological test with CT and MRI was poor, with (Kappa $=0.073,95 \% \mathrm{Cl}: 0.053-1.084)$ and $(\mathrm{Kappa}=0.112,95 \% \mathrm{Cl}: 0.092$ 1.092) and a percentage of agreement of $42.0 \%$ and $45.7 \%$ respectively. Conclusions: Differentiated use and poor concordance between the WB serological test and radiological imaging are performed in patients with a diagnosis of neurocysticercosis in the studied population.

Keywords: Neurocysticercosis, Diagnostic Imaging, Serologic Tests, Diagnostic Techniques and Procedures. (Source: DeCSBIREME).

\section{INTRODUCCIÓN}

La neurocisticerosis (NCC) es una infección parasitaria del sistema nervioso central (SNC), causada por la larva de la solitaria Taenia solium (cisticerco) ${ }^{(1)}$. La teniasis intestinal es la infección parasitaria producida por el estadio adulto de $T$. solium y T. saginata ${ }^{(2)}$; y es la parasitosis más común en países en vías de desarrollo ${ }^{(1)}$, con un estimado de 50 millones de personas infectados alrededor del mundo y 50 mil muertes anuales. La predilección de T. solium por el SNC convierte a la NCC en una de las enfermedades más frecuentes de la práctica neurológica ${ }^{(3)}$. Las manifestaciones clínicas más frecuentes son: cefalea, convulsiones y signos neurológicos de focalización e hipertensión endocraneal ${ }^{(4)}$.

El diagnóstico de la NCC es difíci ${ }^{(5)}$, pues depende de criterios clínicos y epidemiológicos, además de la neuroimagen y confirmación serológica. A pesar que estos métodos son confiables al momento de dar un resultado, aún puede ser un desafío debido a la baja especificidad que se encuentra en los hallazgos clínicos y también a que las pruebas de inmunodiagnóstico presentan valores predictivos poco óptimos, particularmente en entornos endémicos ${ }^{(5)}$.

La neuroimagen es una herramienta para el diagnóstico de NCC, la tomografía axial computarizada (TAC) permite ver lesiones calcificadas y la resonancia magnética (RM) es sensible para diagnosticar NCC parenquimatoso y extraparenquimatoso ${ }^{(6,7)}$. El diagnóstico por imagen no siempre es definitivo. El diagnóstico por western Blot (WB) presenta una especificidad de $100 \%$ y una sensibilidad de $98 \%$ para el diagnóstico de NCC teniendo como criterio de positividad el reconocimiento de uno o más péptidos antigénicos del líquido vesicular: 13, 14, 17, 18, 23, 24, 31, 35 $\mathrm{kDa}$ por anticuerpos específicos presentes en el suero del paciente ${ }^{(8)}$. Cabe destacar que, para la NCC, el WB es sensible a la respuesta de anticuerpos detectables, y puede disminuir su capacidad diagnóstica en casos de lesiones únicas o no viables en el SNC ${ }^{(9,10)}$.

En un estudio previo describió el uso de dos métodos por imágenes (TAC y RM), y uno inmunológico (WB) para el diagnóstico para NCC, reportando que, 13 de 96 casos de NCC fueron diagnosticados con los tres métodos mencionados. Asimismo, observaron estadios diferentes del parásito, ocho de ellos en el estadio vesicular y cuatro en el estadio coloidal. No obstante, sin aplicar la prueba de Western Blot a 50 pacientes se les diagnosticó por imágenes otros estadios que fueron el nodular y el calcificado ${ }^{(11)}$.
El Hospital Regional Lambayeque es un hospital de alta complejidad que recibe pacientes referidos de varias regiones aledañas, pacientes que incluyen aquellos con NCC; sin embargo, el uso de las pruebas radiológicas y serológicas como apoyo al diagnóstico de la NCC por parte de los especialistas aún no se ha estandarizado. Por tal motivo, el presente estudio describió el uso y la concordancia de la imagen radiológica (TAC y RM), con la serología por WB, ambos como pruebas de apoyo al diagnóstico de la NCC en un hospital del norte del Perú.

\section{MATERIAL Y MÉTODOS}

\section{Diseño de investigación}

Se realizó un estudio observacional, retrospectivo tipo pruebas diagnósticas. La población estuvo constituida por pacientes con diagnóstico clínico de NCC, según CIE10-B69, realizado por el médico de turno en emergencia o consulta externa, atendidos en el Hospital Regional Lambayeque (HRL), durante el 2015 a 2017, Chiclayo, Perú. El estudio fue censal.

La unidad de análisis fueron las historias clínicas de los pacientes diagnosticados. Se excluyeron las historias clínicas extraviadas, incompletas o ilegibles.

\section{Recolección de datos}

Los pacientes positivos fueron buscados en el registro informático de la oficina de epidemiología y el registro de análisis del Laboratorio de Parasitología, Metaxénicas y Zoonosis del mencionado hospital. Obtenido los números de historia clínica, la recolección de datos clínicos se realizó mediante el análisis documental de las historias clínicas, previa autorización de acceso al archivo. Se recolectaron los siguientes datos: Resultados a las pruebas de WB, RM y TAC, atendidos en los diferentes servicios del hospital mencionado, además del tipo de lesión, presencia de cefalea y convulsiones, lesión focal, fiebre, recuento de leucocitos y porcentaje de eosinófilos.

En el laboratorio, el estudio serológico mediante WB se realizó para buscar la presencia de anticuerpos contra ocho proteínas vesiculares del parásito: 13, 14, 17, 18, 23, 24, 31, $35 \mathrm{kDa}$. El informe consideró positivo a WB cuando se observó reacción positiva a una o más de las proteínas previamente descritas. Cabe aclarar que el kit comercial usado por el laboratorio fue el Cistiblot $®$ con valores se sensibilidad de 98 $\%$ y especificidad de $100 \%$. Sumado a ello, el estudio de neuroimagen fue registrado y fue informado por un médico 
radiólogo quien independiente del resultado del WB (y viceversa) emitió su resultado. Además, se consideró a la TAC y la RM por separado.

\section{Análisis de datos}

Con los datos obtenidos se elaboraron tablas de resumen y se estimaron frecuencias absolutas y relativas de las características clínicas en relación a la infección por la larva de T. solium. Asimismo, la edad es presentada como media y desviación estándar por su distribución normal evaluado por descriptivos y gráfica. La concordancia entre el WB y la neuroimagen se hizo aplicando el coeficiente de kappa y porcentaje de concordancia. Se utilizó el programa estadístico STATA v 14.0.

\section{Aspectos éticos}

El protocolo y estudio fue revisado y aprobado por el Comité de Ética en Investigación del HRL. La confidencialidad de la información fue garantizada mediante la restricción del acceso a la base de datos solo a miembros del equipo de investigación.

\section{RESULTADOS}

De las 91 historias clínicas evaluadas que mostraban grados de certeza probables de diagnóstico de NCC, solo 67 cumplían con los criterios diagnósticos definitivos para NCC, donde 37 $(55,2 \%)$ correspondieron a pacientes varones. La media de edad fue de 40,2 $( \pm 17,8)$ años. Para el caso de los exámenes por imágenes, se observó que a $59(88,1 \%)$ pacientes se les tomó la TAC, de los cuales, $15(25,4 \%)$ presentaron lesión quística con escólex; además, a $54(80,6 \%)$ pacientes se les tomó la RM, de los cuales, $16(29,6 \%)$ dieron positivo a lesión quística con escólex. Por otro lado, se evidenció que a 39 $(58,2 \%)$ pacientes se les realizó WB, siendo positivos un total de 19 (35,9\%). Ver tabla 1.

Tabla 1. Características generales y diagnósticas de pacientes con neurocisticercosis del Hospital Regional Lambayeque.

\begin{tabular}{|c|c|c|}
\hline Variable & $\mathrm{N}$ & $\%$ \\
\hline \multicolumn{3}{|l|}{ Sexo $(n=67)$} \\
\hline Masculino & 37 & 55,2 \\
\hline Femenino & 30 & 44,8 \\
\hline \multicolumn{3}{|l|}{ Serología por WB $(n=39)$} \\
\hline Positivo & 14 & 35,9 \\
\hline Negativo & 25 & 64,1 \\
\hline \multicolumn{3}{|l|}{ Imagen radiología por TAC ( $\mathrm{n}=59)$} \\
\hline Lesión quística con escólex (Positivo) & 15 & 25,4 \\
\hline Lesión con quiste vesicular (Positivo) & 2 & 3,4 \\
\hline Lesión nodular (Positivo) & 32 & 54,2 \\
\hline Lesión multilobulada (Positivo) & 4 & 6,8 \\
\hline Lesión ventricular hipodensa (Positivo) & 1 & 1,7 \\
\hline Aspecto normal (Negativo) & 5 & 8,5 \\
\hline \multicolumn{3}{|l|}{ Imagen radiología por RM ( $n=54)$} \\
\hline Lesión quística con escólex (Positivo) & 16 & 29,6 \\
\hline Lesión con quiste vesicular (Positivo) & 1 & 1,9 \\
\hline Lesión nodular (Positivo) & 22 & 40,7 \\
\hline Lesión multilobulada (Positivo) & 4 & 7,4 \\
\hline Aspecto normal (Negativo) & 11 & 20,4 \\
\hline \multicolumn{3}{|l|}{ Imagen radiológica por TAC y $R M(n=30)$} \\
\hline Lesión quística con escólex (Positivo) & 10 & 33,3 \\
\hline Lesión multilobulada (Positivo) & 3 & 10 \\
\hline Lesión nodular (Positivo) & 17 & 56,7 \\
\hline
\end{tabular}

Las características clínicas al momento de ingreso de los pacientes se encontraron que $51(76,1 \%)$ tuvieron cefalea y $42(62,7 \%)$ presentaron convulsiones, más información se reporta en la Tabla 2. Para el caso de la concordancia observada entre la prueba serológica por WB y el examen por imagen mediante TAC fue baja (Kappa=0,073, IC95\%: 0,053 $1,084)$, ver tabla 3; asimismo, la concordancia observada entre la prueba serológica por WB y el examen por imagen mediante RM también fue baja (Kappa=0,112, IC95\%: 0,092 $1,092)$, ver tabla 4.

Tabla 2. Características clínico-laboratoriales de pacientes con neurocisticercosis del Hospital Regional Lambayeque.

\begin{tabular}{lcc} 
Variable & $N$ & $\%$ \\
Cefalea $(n=67)$ & 51 & 76,1 \\
$\quad$ Sí & 16 & 23,9 \\
$\quad$ No & & \\
Convulsiones $(n=67)$ & 42 & 62,7 \\
$\quad$ Sí & 25 & 37,3 \\
$\quad$ No & & \\
Fiebre $(n=67)$ & 3 & 4,5 \\
$\quad$ Sí & 64 & 95,5 \\
$\quad$ No & & \\
Leucocitosis $(n=42)$ & 18 & 42,9 \\
$\quad$ Sí & 24 & 57,1 \\
$\quad$ No & & \\
Eosinofilia $(n=42)$ & 6 & 14,3 \\
$\quad$ Sí & 36 & 85,7 \\
$\quad$ No & & \\
\hline
\end{tabular}

Tabla 3. Concordancia entre la prueba serológica por WB con el examen imagenológico por TAC, en pacientes con diagnóstico de neurocisticercosis en el Hospital Regional Lambayeque $(\mathrm{N}=31)$.

\begin{tabular}{lccccc} 
Western Blot & \multicolumn{2}{c}{ TAC } & & $\begin{array}{c}\% \text { de } \\
\text { acuerdo }\end{array}$ & Coeficiente Kappa (IC: 95\%) \\
\cline { 2 - 3 } & Positivo & Negativo & & \\
Positivo & 11 & 0 & 42 & $0,073(0,053-1,084)$ \\
Negativo & 18 & 2 & & \\
\hline
\end{tabular}

Tabla 4. Concordancia entre la prueba serológica por WB con el examen imagenológico por RM, en pacientes con diagnóstico de neurocisticercosis en el Hospital Regional Lambayeque $(\mathrm{N}=35)$.

\begin{tabular}{|c|c|c|c|c|}
\hline \multirow{2}{*}{ Western Blot - } & \multicolumn{2}{|c|}{ RM } & \multirow{2}{*}{$\begin{array}{c}\% \text { de } \\
\text { acuerdo }\end{array}$} & \multirow{2}{*}{ Coeficiente Kappa (IC: 95\%) } \\
\hline & Positivo & Negativo & & \\
\hline Positivo & 10 & 1 & \multirow{2}{*}{45,7} & \multirow{2}{*}{$0,112(0,092-1,092)$} \\
\hline Negativo & 18 & 6 & & \\
\hline
\end{tabular}

\section{DISCUSIÓN}

La NCC afectó en nuestro caso tanto a pacientes varones y mujeres en aproximadamente $50 \%$ cada uno, esto puede ser diferente en proporciones, como en Korea donde los pacientes del sexo masculino fueron los mayores afectados con un $79 \%^{(12)}$, pero es más frecuente que sean proporciones similares, como un estudio hecho en el sur de India, indicó un $52 \%$ de casos positivos para varones ${ }^{(13)}$ y en Lima, Perú, encontrando que el $56 \%$ de los pacientes que padecían NCC fueron mujeres ${ }^{(14)}$, por lo tanto, esta enfermedad puede afectar tanto a hombres como a mujeres en igual proporción. Analizando la prueba de Western Blot, en nuestro trabajo 
solo fue positivo para NCC en un $35,9 \%$ de los 39 casos analizados a quiénes se les solicitó la prueba, menor a lo reportado en Londres, Inglaterra, que tomaron datos de pacientes en un periodo de 15 años. En este estudio, el examen de WB mostró seropositividad en 52\% de todos los casos presentados ${ }^{(15)}$. Asimismo, otro estudio originario de Perú donde la mayoría de pacientes mostraron resultados positivos para NCC con esta prueba, hasta un $85 \%^{(14,16)}$.

Además, cabe destacar que, en un estudio se reportó que de 300 muestras comunitarias de individuos con síntomas de NCC, siendo las epilepsias el más común y los lugares de residencia de dichas personas era endémico de la enfermedad, solamente $39(13 \%)$ dieron positivo para WB ${ }^{(17)}$, lo cual es distante con el presente estudio que resultó casi $36 \%$ de positividad para WB, lo cual sugiere que al momento de evaluar a pacientes nosocomiales y comunitarios se debe tomar criterios de síntomas y exámenes por imágenes adicionales a pruebas serológicas.

En cuanto a las características clínicas la mayoría de los pacientes presentaron cefalea, tal y como lo mencionan estudios realizados en Korea $^{(12)}$ y Ecuador ${ }^{(18)}$ donde la cefalea fue el síntoma clínico más común en los casos estudiados de NCC, no obstante, en una investigación donde trataban de comparar la resonancia magnética para diferenciar diagnostico entre NCC y tuberculomas, mencionaban que en los pacientes con NCC el síntoma clínico más frecuente eran las convulsiones y el no muy común era la cefalea ${ }^{(19)}$. Estos síntomas son los más reportados a nivel mundial ${ }^{\left({ }^{(15)}\right.}$, por lo que debería complementar el diagnóstico de NCC con pruebas de imagen y de laboratorio cuando ingresen por cefaleas, convulsiones y procedan de zonas endémicas como el norte del Perú ${ }^{(4)}$.

El análisis de concordancia entre el WB y los exámenes por imágenes mediante el test de Kappa, se encontró escaso acuerdo; este hallazgo puede explicarse por los diferentes rendimientos entre las pruebas, por el diferente y heterogéneo uso que los médicos le dan; y tal vez, debido a lesiones únicas donde el WB pierde rendimiento diagnóstico. En contraposición a lo que se menciona, en un estudio realizado en México, donde se comparó la RM con la técnica serológica de ELISA modificado, tanto para muestras de suero como en fluidos cerebroespinal, donde reportaron alta concordancia en el caso de las muestras de fluido y acuerdo moderado para las muestras de suero(20).

Existe pocos de estudios que comparen pruebas diagnósticas tipo serología e imágenes, sin embargo, hay evidencia de que se están probando pruebas de ELISA modificados y PCR para tratar de diagnosticar esta enfermedad e incluso se ha comparado con exámenes radiológicos, lo cual sugiere un mayor esfuerzo para poder establecer un diagnostico apropiado para la NCC.

En el presente estudio los porcentajes de las imágenes radiológicas y WB positivos son diferentes y bajos respecto al total de diagnósticos de NCC. Este hallazgo se podría explicar por el escaso uso de los criterios de del Brutto para el correcto diagnóstico de la NCC, principalmente en algunos médicos no especialistas en neurología. A saber, en la NCC parenquimatosa, la TC y RM presentan como hallazgos más característicos las lesiones quísticas bien definidas y las calcificaciones puntiformes múltiples; para la NCC meníngea, se suele revelar hidrocefalia, captación anormal del contraste en las leptomeninges basales, quistes subaracnoideos e infartos cerebrales. La TC es la técnica elegible para el estudio de casos probables de NCC, mientras que la RM debe reservarse para aquellos pacientes con TC normal o en los que el aspecto tomográfico de las lesiones no sea concluyente $e^{(4)}$.

El presente estudio tuvo como limitación la fuente secundaria de la información (historia clínica) que pudo haber llevado a un sesgo de medición (cuando los médicos no solicitaban la prueba serológica al ver resultados positivos a la imagen radiológica). Asimismo, la alta frecuencia de información incompleta en las historias clínicas no permitió tener la cantidad y calidad esperados.

En conclusión, se encontró un uso diferenciado y escaza concordancia entre la prueba serológica por Western Blot e imagen radiológica por tomografía y resonancia en el diagnóstico de la neurocisticercosis de pacientes de un hospital del norte del Perú, 2015 a 2017.

\section{REFERENCIAS BIBLIOGRÁFICAS}

1. Ballón B. Neurocisticercosis racemosa. Rev Exp en Med [Internet].

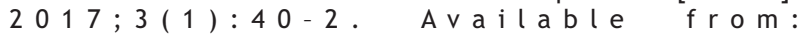
http://rem.hrlamb.gob.pe/index.php/REM/article/view/81/76

2. Velasquez $R$, Rojas $S$, Briceño $A$, Prieto $M$. Neurocisticercosis: Enfermedad infecciosa desatendida, olvidada y emergente. A propósito de un caso. Comunidad y Salud [Internet]. $2016 ; 14(2): 14-23$. A va i la ble f rom: http://www.redalyc.org/pdf/3757/375749517003.pdf

3. Serrano G, Ortíz J, Ochoa I. Neurocisticercosis. Presentación de un caso. Medisur [Internet]. 2009;7(2):54-7. Available from: http://scielo.sld.cu/pdf/ms/v7n2/v7n2a507.pdf

4. del Brutto 0 . Neurocisticercosis. Rev Neurol [Internet]. $1999 ; 29$ ( 5 ): $456-66$. A v a i l a ble from: https://www.neurologia.com/articulo/99313

5. Garcia H, Nash T, del Brutto O. Clinical symptoms, diagnosis, and treatment of neurocysticercosis. Lancet Neurol. 2014;13(12):1202-15.

6. García $\mathrm{HH}$, Del Brutto $\mathrm{OH}$. Imaging findings in neurocysticercosis. Acta Trop [Internet]. 2003;87(1):71-8. Available from: https://scihub.tw/10.1016/s0001-706x(03)00057-3

7. Zea-Vera A, Cordova EG, Rodriguez S, Gonzales I, Pretell EJ, Castillo $\mathrm{Y}$, et al. Parasite antigen in serum predicts the presence of viable brain parasites in patients with apparently calcified cysticercosis only. Clin Infect Dis [Internet]. 2013;57(7):154-9. Available from: https: //sci-hub.tw/10.1093/cid/cit422

8. Sánchez EL, Náquira C, Vega S, Miranda EF, Quispe WM, Ayala-Sulca ER. Manual de procedimientos para el diagnóstico serológico de las zoonosis parasitarias. [Internet]. $32^{\circ}$. INS. Lima: Instituto Nacional de Salud; 2020.112 p. Available from: http://bvs.minsa.gob.pe/local/minsa/1585.pdf

9. Noh J, Rodriguez S, Lee YM, Handali S, Gonzalez AE, Gilman RH, et al. Recombinant protein- and synthetic peptide-based immunoblot test for diagnosis of neurocysticercosis. J Clin Microbiol [Internet]. 2014;52(5):1429-34. Available from: https://scihub.tw/10.1128/JCM.03260-13

10. Ayala-Sulca E, Miranda-Ulloa E. Evauación de electroinmunotransferencia utilizando antígeno mix nativo purificado de líquido de cisticerco de Taenia solium para el diagnóstico de cisticercosis humana. Rev Peru Med Exp Salud Publica [Internet]. 2015;32(3):485-91. Available from: http://www.scielo.org.pe/pdf/rins/v32n3/a11v32n3.pdf

11. Rojas-Panta G, Toro-Huamanchumo C, Altamirano-Mego E. Perfil clínico y epidemiológico de los pacientes con diagnóstico de neurocisticercosis en dos hospitales de Chiclayo, Perú. Rev Horiz Médico [Internet]. 2017;17(1):11-7. Available from: 
http://www.horizontemedicina.usmp.edu.pe/index.php/horizonte med/article/view/548/365

12. Son $\mathrm{H}-\mathrm{J}$, Kim MJ, Jung $\mathrm{KH}$, Choi S, Jung J, Chong YP, et al. Neurocysticercosis: Clinical Characteristics and Changes from 26 Years of Experience in an University Hospital in Korea. Korean J Parasitol. 2019;57(3):265-71.

13. Murthy JMK, Seshadri V. Prevalence, clinical characteristics, and seizure outcomes of epilepsy due to calcific clinical stage of neurocysticercosis: Study in a rural community in south India. Epilepsy Behav [Internet]. 2019;98:168-72. Available from: https://doi.org/10.1016/j.yebeh.2019.07.024

14. Arroyo G, Rodriguez S, Lescano AG, Atroy KA, Bustos JA, Santivañez S, et al. Antibody Banding Patterns of the Enzyme-Linked Immunoelectrotransfer Blot and Brain Imaging Findings in Patients with Neurocysticercosis. Clin Infect Dis. 2018;66(2):282-8.

15. Hunter E, Cliff $M$, Armstrong $M$, Manji H, Jäger HR, Chiodini $P$, et al. Active neurocysticercosis at the Hospital for Tropical Diseases, London: A clinical case series. Trans R Soc Trop Med Hyg. 2018;112(7):326-34

16. Garcia HH, Castillo Y, Gonzales I, Bustos JA, Saavedra H, Jacob L, et al. Low sensitivity and frequent cross-reactions in commercially available antibody detection ELISA assays for Taenia solium cysticercosis. Trop Med Int Heal [Internet]. 2018;23(1):101-5. A v a i l a ble f $\mathrm{r}$ o m : https://www.ncbi.nlm.nih.gov/pmc/articles/PMC5760338/pdf/nih ms921439.pdf

17. Alarakol SP, Joloba ML, Aginya EO. Seroprevalence of Taenia solium cysticercosis among people with epilepsy epileptic patients in three rural districts of Northern Uganda. J Parasitol Vector Biol. 2017;9(5):47-56.

18. Romo ML, Carpio A, Parkhouse RME, Cortéz MM, Rodríguez-Hidalgo R. Comparison of complementary diagnostic tests in cerebrospinal fluid and serum for neurocysticercosis. Heliyon. 2018;4(12):1-9.

19. Ghosh RN, Vyas S, Singh P, Khandelwal N, Sankhyan N, Singhi P. Perfusion magnetic resonance imaging in differentiation of neurocysticercosis and tuberculoma. Neuroradiology. 2019;61(3):257-63.

20. Fleury A, Garcia E, Hernández M, Carrillo R, Govezensky T, Fragoso G, et al. Neurocysticercosis: HP10 Antigen Detection Is Useful for the Follow-up of the Severe Patients. PLoS Negl Trop Dis. 2013;7(3):1-7. 